\title{
SUPERIOR LIMBIC KERATOCONJUNCTIVITIS OF THEODORE: A CASE REPORT AND LITERATURE REVIEW
}

\section{F Carbonaro, SHO; E Khadem, Consultant; Ophthalmology Furness General Hospital}

This is a case report of a 35-year-old male with superior limbic keratoconjunctivitis (SLK) of Theodore. This is a disorder which normally presents in women aged between 20 and 60 years, who may have associated thyroid disease. The clinical presentation of this case and its management are presented.

An introduction is given on when the disorder was described and by whom. A discussion on the usual presentation and its associations follows, together with investigations carried out and current treatment methods. Theories on its pathogenesis are also discussed.

\section{INTRODUCTION}

In 1953 Braley and Alexander described some cases of superficial punctuate keratitis with filaments, which were probably examples of superficial punctuate keratitis ${ }^{(1)}$. In 1961 Theodore published a letter ${ }^{(2)}$ on ten of his patients with findings which are now known to be SLK. He also suggested that these cases could represent a clinical entity, possibly with a viral origin.

Thygeson and Kimura described 17 cases $^{(3)}$ whose characteristics and laboratory findings were typical of SLK. Theodore later discussed their paper and outlined in detail the characteristics of his own similar cases, which he named 'superior limbic keratoconjunctivitis'. In 1963 Theodore published the first definitive paper on the subject ${ }^{(4)}$ giving it its name. Five years later, Tenzel and Corwin reported an association between thyroid abnormalities and SLK. A mimicking disorder has also been reported in soft contact lens wearers, especially with thimerosal-preserved solutions ${ }^{6^{5}}$

Over $50 \%$ of cases of SLK are associated with keratoconjunctivitis sicca ${ }^{(6)}$. The global incidence of this disorder is as yet unknown.

The case we encountered was unusual in that the patient was male, with no other medical disorders.

\section{CASE PRESENTATION}

A 35-year-old man presented to Furness General Hospital with a $1 \frac{1}{2}$ year history of bilateral sore and itchy eyes, with foreign body sensation and excessive lacrimation and mucoid discharge. The symptoms were worse in the right than the left eye. He had no other medical history and was not on any medication.

On examination he was found to have a visual acuity of $6 / 5$ in both eyes.
Slit lamp examination showed fine papillary hypertrophy of the conjunctival tarsal plate, hyperaemia of the superior bulbar conjunctiva and superior limbic papillary hypertrophy (Figures 1 and 2). The cornea and anterior chamber were fine, as were his intra-ocular pressures.

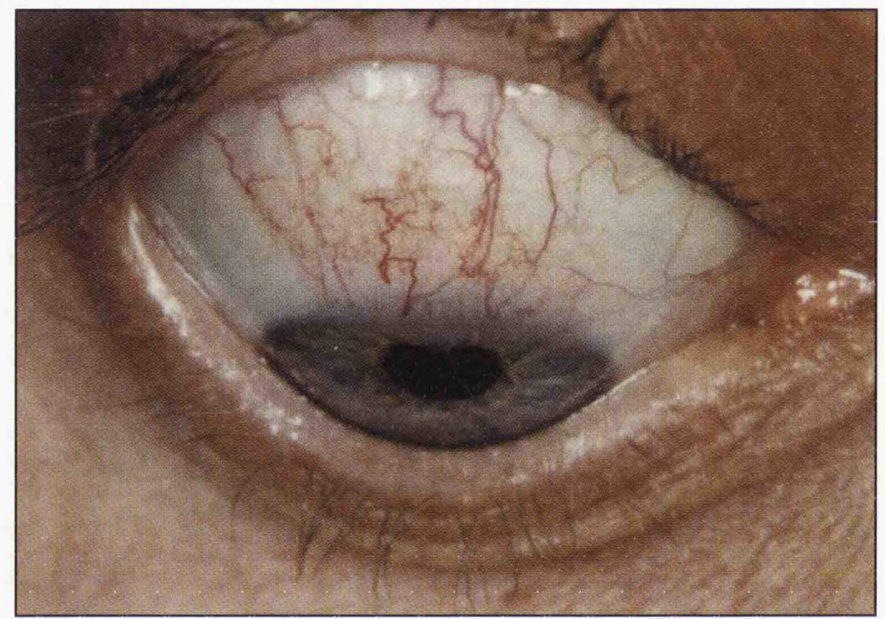

Figure I Right eye, showing inflammation of superior bulbar conjunctiva

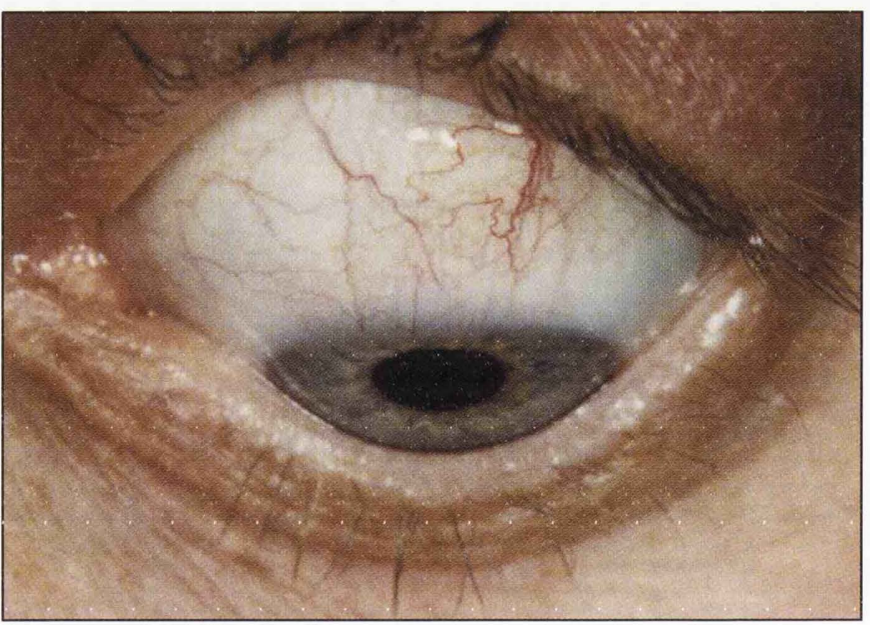

Figure 2 Left eye, showing inflammation of superior bulbar conjunctiva

He was started on Nedocromil Sodium (Rapitil) drops and Fluorometholone (Allergan) drops and we reviewed him six weeks later. The symptoms improved when he was taking the Fluorometholone (Allergan) but as this was tailed off they exacerbated; he was therefore re-started on them and thyroid function tests were requested. His thyroid tests came out within normal range and he was switched to Prednisolone (Predforte) drops, which he is currently on.

On his last appointment, at the outpatient clinic, he reported that when on holiday in Scotland for a week he stopped taking the drops and was completely free of 
symptoms for the duration of his stay there. He also informed us that his symptoms more or less coincided with him having a pet rabbit and we therefore requested an allergen test.

\section{DISCUSSION}

SLK of Theodore is an uncommon, chronic inflammatory disorder most commonly seen in middle-aged women, often with associated thyroid disease ${ }^{(7)}$. The condition is usually bilateral, but may be asymmetrical. It follows a prolonged course, with exacerbations and remissions and eventual resolution without sequelae. Individual episodes may persist from days to more than a year, with remissions lasting from a few weeks to the rest of the patient's life.

The exact pathophysiology is unclear. Infectious agents have been ruled out due to routinely negative cultures and the fact that antimicrobial therapy is of no value. An autoimmune aetiology has been postulated due to the nature of the condition (exacerbations and remissions), female predominance and the association with thyroid and other autoimmune disorders.

Wright ${ }^{(8)}$ postulated a mechanical cause, noting that most patients appear to have loose and redundant superior bulbar conjunctiva, which often rubs against the superior palpebral conjunctiva and limbus. Factors such as tight upper eyelids, prominent globes, and thyroid eye disease might act to initiate tissue contact and conjunctival redundancy. A newer theory regarding the aetiology of SLK implicates a local tear deficiency to the superior keratoconjunctiva.

Typical presentation is with exacerbations and remissions of burning, irritated and red, watering eyes ${ }^{(9)}$. Examination of the eyes using the slit lamp reveals a sectoral inflammation and injection of the superior bulbar conjunctiva. The inflammation is most prominent near the limbus and fades towards the upper fornix. Eversion of the upper eyelid reveals a uniform papillary hypertrophy along the tarsus. Filaments are encountered within the precorneal tear film in roughly $50 \%$ of all patients with SLK.

Diagnosis is made on a clinical basis, although blood investigations of thyroid function should be carried out and a Schirmer test is useful. Staining the eye with fluoroscein and rose bengal is also necessary for making a diagnosis. The only laboratory test that is predictably positive, is the finding of keratinized epithelial cells in scrapings from the conjunctival tissue.

Treatment is aimed at altering the abnormal mechanical interaction between the upper lid and the superior limbus. Tear substitutes to lubricate the eye are available in both droplet and gel form and are often of use in relieving discomfort, albeit temporarily. Mast cell stabilizers like Cromolyn sodium $4 \%$ are also of use ${ }^{(10)}$. Silver nitrate
$(0.1-0.5 \%)$ solution is the treatment of choice in most cases, causing chemical cauterization of the irregular tissue. Soft bandage contact lenses are also very useful in relieving the symptoms ${ }^{(5)}$.

There are also surgical methods available, namely thermocauterizaton of the superior bulbar conjunctiva; occlusion of the puncta, through which the tears drain and, as a last resort, rescetion of the superior limbal conjunctiva.

The main problem in the diagnosis of this condition is the practitioner's lack of awareness of its existence. Unfortunately, it is not uncommon for a patient to go through various remedies without success, before they are diagnosed with SLK and the proper treatment given.

The prognosis is excellent and as mentioned earlier the condition resolves spontaneously after a prolonged course of treatment.

\section{REFERENCES}

1 Nelson JD. Superior limbic keratoconjunctivitis (SLK). Eye 1989;3(2):180-90

2 Theodore FH. The Collected Letters of the International Correspondence Society of Ophthalmology 1961;6:1989

3 Thygeson P, Kimura SJ. Chronic conjunctivitis. Trans Am Acad Ophthalmol 1963;67:494

4 Theodore FH. Further observations on superior limbic keratoconjunctivitis. Trans Am Acad Ophthalmol Otolaryngol 1967;71(2):341-51

5 Sendele DD, Kenyon KR, Mobilia EF et al. Superior limbic keratoconjunctivitis in contact lens wearers. Ophthalmol 1983;90(6):616-22

6 Watson S, Tullo AB, Carley F. Treatment of superior limbic keratoconjunctivitis with a unilateral bandage contact lens. $\mathrm{Br}$ J Ophthalmol 2002;86(4):485-6

7 Kadrmas EF, Bartley GB. Superior limbic keratoconjunctivitis. A prognostic sign for severe Graves ophthalmopathy. Ophthalmol 1995;102(10):1472-5

8 Wright P. Superior limbic keratoconjunctivitis. Trans Ophthalmol Soc UK 1972;92:555-560

9 Wilson FM 2nd, Ostler HB. Superior limbic keratoconjunctivitis. Int Ophthalmol Clin 1986;26(4):99-112

10 Confino J, Brown SI. Treatment of superior limbic keratoconjunctivitis with topical cromolyn sodium. Ann Ophthalmol 1987;19(4):129-31 